\title{
MAJOR H $\alpha$ FLARES IN CENTERS OF ACTIVITY WITH VERY SMALL OR NO SPOTS
}

\author{
HELEN W. DODSON and E. RUTH HEDEMAN \\ McMath-Hulbert Observatory, The University of Michigan, U.S.A.
}

(Received 3 February, 1970)

\begin{abstract}
Major $\mathrm{H} \alpha$ flares (importance $>2$ ) in plages with only small or no spots constitute a rare but well observed aspect of solar activity. Information relating to 83 such flares has been assembled and studied. In the years 1956-1968 these flares represented $\sim 7 \%$ of all confirmed flares of importance $>2$. In general, the flares were of unusually long duration and rose to maximum intensity slowly. A flash phase was often absent or poorly defined. In a number of cases, the flare emission included two bright filaments more or less parallel. The flares usually occurred during the late, flare-poor phase of a center of activity, and their outbreak did not presage a resurgence of activity in subsequent rotations. The flares were frequently associated with the position of dark filaments.

Like major flares in general, the flares in regions with small or no spots usually were associated with long-enduring radiation (gradual rise and fall and/or postburst increase) at $\sim 10 \mathrm{~cm}$, and with $\mathrm{X}$-ray enhancements $(2-12 \AA)$ at least as great as 4 times the quiet Sun. They were deficient, in the associated occurrence of strong, impulsive, centimetric bursts and of X-ray events $>20$ times the quiet Sun. The absence of large spots apparently did not inhibit the occurrence of Type II bursts.

Only $41 \%$ of the major flares here studied were accompanied by shortwave fades and of these ionospheric disturbances only a few were great events. In general the flares were not followed by the detection of high energy particles or the onset of geomagnetic storms. However, a few of the flares (including those of 1967 January 11 and February 13) apparently were associated with well observed particle emission and suggest that the presence of a large complex spot is not always necessary for the acceleration of energetic particles or the emission of solar plasma at the time of a large $\mathrm{H} \alpha$ flare.
\end{abstract}

\section{Introduction}

In general, $\mathrm{H} \alpha$ flares of importance 2 and 3 occur during the flare-rich, mature phase of centers of activity at times when large, well developed spots and strong, complex magnetic fields are present. There are, however, well documented observations of major $\mathrm{H} \alpha$ flares in concomitantly spotless and nearly spotless regions. Although such flares are infrequent, full realization of their occurrence, and information relating to their characteristics, may be of value for theoretical studies which are directed towards an understanding of the physical circumstances necessary and sufficient for the outbreak of large $\mathrm{H} \alpha$ flares.

Published material shows that it has been known for many years that $\mathrm{H} \alpha$ flares, even important flares, occasionally occur in spotless regions. As early as 1935, H.W. Newton described two flares, both evaluated as importance 2 in the Quarterly Bulletin on Solar Activity, and indicated that one was in a spotless region and that the other was associated with only a small group of spots. In 1936 the Quarterly Bulletin included the remark "Pas de tache dans cette région active" in connection with a flare of importance 2 observed at Mount Wilson on October 12 of that year. A flare of importance 3 observed at Meudon and Greenwich on 1936 December 30 at N17 W56 was in a region with a spot whose area was only 50 millionths of the solar hemisphere on the day of the flare. For a picture of this flare see Figure 1a. More impor- 


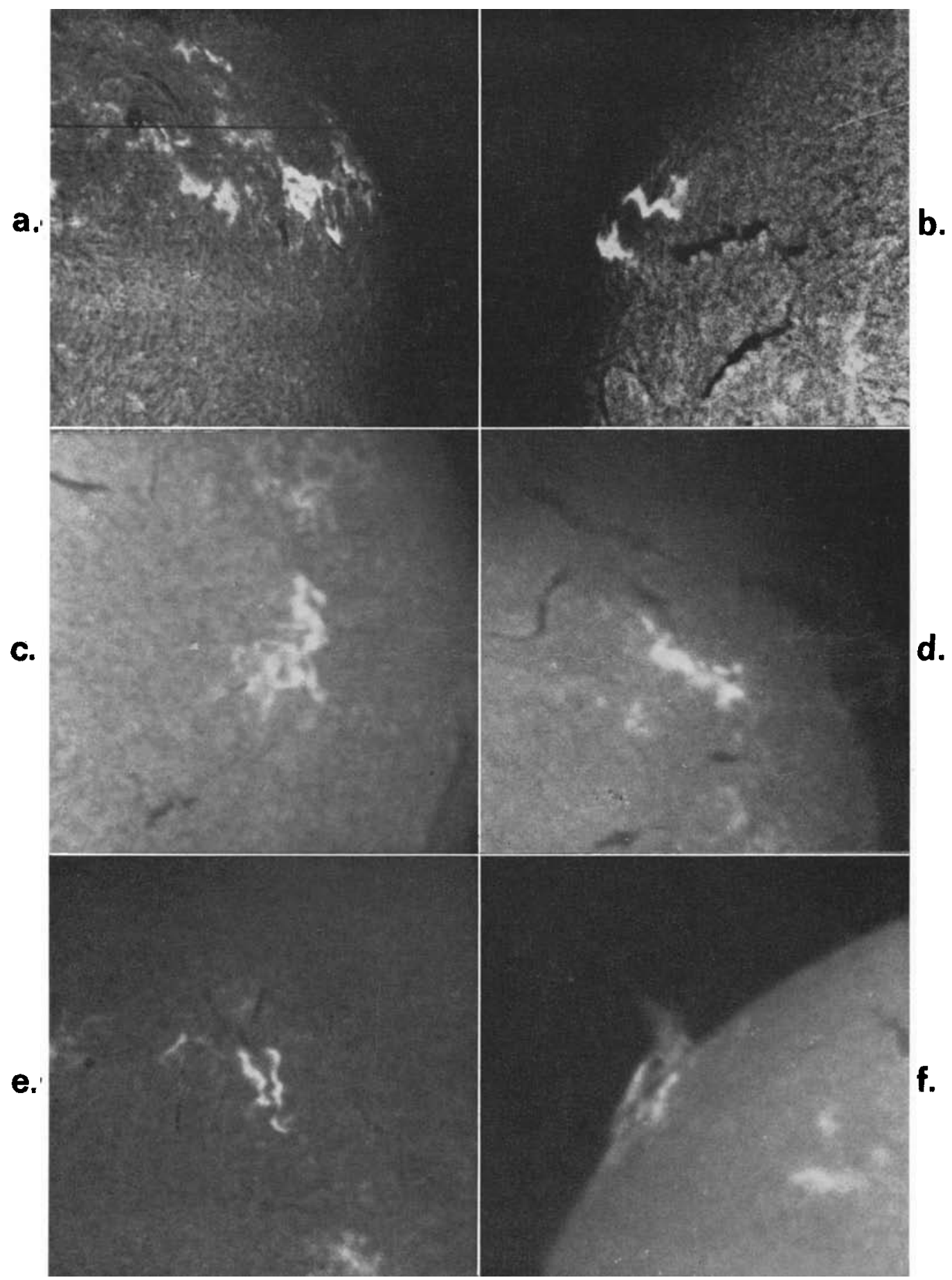

Fig. 1. H $\alpha$ pictures showing examples of major flares in regions with small or no spots (Table I). (a) Flare No. 6: 1936 Dec. 30d $10^{\mathrm{h}} 58^{\mathrm{m}}$ UT (Meudon); (b) Flare No. $21: 1957$ Nov. $29^{\mathrm{d}} 02^{\mathrm{h}} 14^{\mathrm{m}}$ UT $^{\mathrm{T}}$ (Sydney); (c) Flare No. 31: 1958 Aug. 30 $20^{\mathrm{h}} 21^{\mathrm{m}}$ UT (McMath-Hulbert); (d) Flare No. $38: 1959$ Feb. $2^{\mathrm{d}} 10^{\mathrm{h}} 37^{\mathrm{m}}$ UT (Meudon); (e) Flare No. 63: 1966 May 25d $11^{\mathrm{h}} 06^{\mathrm{m}}$ UT (McMath-Hulbert); (f) Flare No. 71: 1967 Feb. $6^{\mathrm{d}} 18^{\mathrm{h}} 55^{\mathrm{m}}$ UT (limb event, McMath-Hulbert). 
tantly, in 1944, R. S. Richardson published a study entitled 'Occurrence of Solar Flares where no Sunspot Group was Observed'. For this article Richardson reexamined data relating to 593 flares observed at Mount Wilson from 1917 to 1943 and reported that "after eliminating all doubtful cases, five definite examples remained of flares in the chromosphere where no spot group was recorded in the photosphere". Three of the five cases were evaluated as flares of importance 2. Additionally, Giovanelli in 1939 pointed out a possible increase in flare activity in the final stages of decay of spot groups, at times when spots, if present at all, were very small. More recently Bruzek (1959) and Švestka (1968) have described a major flare in a region with a dying spot, and Hyder (1967) discussed flare-like brightenings near filaments in 'quiet regions' on the solar disk.

Comparison of flare and spot data for the past 30 years provides rare but additional examples of major $\mathrm{H} \alpha$ flares in regions with only very small or no spots. It is often difficult to distinguish between regions with no spots and those with a very small spot because the various observers of sunspots frequently disagree in these matters. The differences in the reports of spots probably result from either different observing conditions at the respective stations or from actual changes with time in a region. In this study a region is considered to have been spotless on a particular day if Zürich, Greenwich, Mount Wilson, and McMath-Hulbert Observatories all recorded no spot on the day in question. A region is considered to have included only a small spot on a certain day if the reported area of the spot on that day was less than 110 millionths on the solar hemisphere.

\section{The Present Survey}

In an attempt to identify major $\mathrm{H} \alpha$ flares in regions with only small or no spots, we have examined spot data associated with all flares of importance $>2$ for the 12-year interval July 1957 to June 1969. In addition to the Mount Wilson, Zürich, and Greenwich Observatory spot measurements we have used the Cartes Synoptiques (Meudon), the Quarterly Bulletin on Solar Activity, and all relevant observations at the McMath-Hulbert Observatory. On the basis of this material we have prepared a list of flares of importance 2 and 3 in spotless regions and in regions with only very small spots. In this work, a special note has been made if the region in question was within $\sim 15^{\circ}$ of another region with a major spot. Cases involving doubtful flare observations or greatly confused spot data have been omitted. The list for the 12 -year interval studied in detail contains 64 entries. See Table I.

For the years prior to 1957.5 we have assembled a partial list of major $\mathrm{H} \alpha$ flares in regions with very small or no spots. The list includes the flares reported by Richardson (1944) and Newton (1935), and also all flares of importance $>2$ for which Zürich and Mount Wilson spot data confirmed remarks in the Quarterly Bulletin, either those by the editor, "Aucune tache n'a été observée à Meudon dans ce centre", or those by individual reporters "Pas de taches visibles dans les alentours." Mme. Martres, at Meudon, has also called several cases to our attention. In this way 19 additional major $\mathrm{H} \alpha$ flares in regions with small or no spots have been identified. 
Information relating to the aforementioned $83 \mathrm{H} \alpha$ flares of importance $>2$ in regions with only small or no spots forms the basis of the present study. Table I provides a partial summary of the data used in the investigation. For 34 of the flares in Table I, the associated regions were spotless and for only 8 cases did the area of the residual spot exceed 50 millionths of the solar hemisphere. The list includes 16 flares of importance 3, and 67 flares of importance 2. There are no obvious peculiarities in the basic flare data. The distribution with respect to Universal Time shows 43 flares during the first half of the Universal Day and 40 during the second half. The distribution of the flares on the solar disk is gratifyingly uniform from 0-69 C.M.D. and there are only 7 flares in the study for which the central meridian distance is $>70^{\circ}$. Therefore, in general, the absence of a spot or the smallness of its area cannot be attributed, for the regions here considered, to difficulties and problems of spot measurements near the solar limb.

TABLE I

$\mathrm{H} \alpha$ flares, impotance $>2$, in regions with only small or no spots

\begin{tabular}{|c|c|c|c|c|c|c|c|c|c|}
\hline \multirow{3}{*}{ No. } & \multirow{3}{*}{ Date } & & \multicolumn{4}{|l|}{$\mathrm{H}_{\alpha}$ Flare } & \multirow{3}{*}{$\begin{array}{l}\text { Spot } \\
\text { Data }^{a}\end{array}$} & \multirow{3}{*}{$\begin{array}{l}\text { SWF }^{b} \\
(1956-69)\end{array}$} & \multirow{3}{*}{$\begin{array}{l}\text { Remarks }^{\mathrm{e}} \\
(1956-1969)\end{array}$} \\
\hline & & & \multicolumn{2}{|l|}{ Time (UT) } & \multirow{2}{*}{ Position } & \multirow[t]{2}{*}{ Imp. } & & & \\
\hline & & & Start End & Max. & & & & & \\
\hline 1 & 1918 & Sept. 15 & 1825 & - & N10 W39 & 2 & A-0 & - & \\
\hline 2 & 1935 & May 3 & 0838 & - & S23 E22 & 2 & B-11 & - & \\
\hline 3 & 1935 & May 10 & $0920-1100$ & - & N23 W50 & 2 & $A-0$ & - & \\
\hline 4 & 1936 & Oct. 12 & $1641-1645$ & - & N20 E15 & 2 & $A-0$ & - & \\
\hline 5 & 1936 & Oct. 12 & $1641-1645$ & - & N18 W02 & 2 & B-102 & - & \\
\hline 6 & 1936 & Dec. 30 & $1053-1250$ & - & N17 W56 & 3 & B-50 & - & \\
\hline 7 & 1937 & July 16 & $2245-2412$ & - & S15 W21 & 3 & $\mathrm{AC}-0$ & - & \\
\hline 8 & 1946 & May 2 & $0635-0645$ & - & S34 W13 & 2 & B-16 & - & \\
\hline 9 & 1946 & Nov. 4 & $0100-0125$ & - & N28 E00 & 2 & B-10 & - & \\
\hline 10 & 1948 & June 7 & $0838-0920$ & 0848 & N17 E50 & 2 & B-28 & - & \\
\hline 11 & 1949 & Mar. 5 & $0835-0925$ & - & N10 E50 & 2 & $\mathrm{BC}-10$ & - & \\
\hline 12 & 1949 & June 12 & 0830-0905 & - & N23 W43 & 2 & $\mathrm{AC}-0$ & - & \\
\hline 13 & 1949 & June 16 & 0903-0930 & - & N23 E54 & 2 & $A-0$ & - & \\
\hline 14 & 1949 & Oct. 24 & $0900-1210$ & 0911 & S22 W47 & $2-$ & $A-0$ & - & \\
\hline 15 & 1956 & Feb. 29 & $2220-2309$ & - & S30W22 & 3 & B-73 & $S / 3$ & $2800 / 525$ \\
\hline 16 & 1956 & July 8 & 0522 & - & S29 E42 & 2 & $A C-0$ & - & $q$ \\
\hline 17 & 1956 & July 25 & $1405-1510$ & - & S12 W15 & 2 & B-12 & - & $\mathrm{q}$ \\
\hline 18 & & Sept. 25 & $1058-1157$ & 1108 & N21 E32 & 2 & A-0 & - & $\mathrm{q}$ \\
\hline 19 & 1956 & Sept. 25 & $1240-1400$ & - & N22.E35 & 2 & $\mathrm{~A}-0$ & - & $q$ \\
\hline 20 & 1957 & July 18 & $2234-2430$ & 2306 & S22 E27 & 2 & $\mathrm{AC}-0$ & - & $\mathrm{q}$ \\
\hline 21 & 1957 & Nov. 29 & $0045-0600$ & 0213 & N41 E63 & $3+$ & $\mathrm{A}-0$ & - & Types II, III \\
\hline 22 & 1957 & Dec. 8 & $0814-0904$ & 0835 & N33 E22 & 2 & $\mathrm{~B}-9$ & - & \\
\hline 23 & 1957 & Dec. 12 & $0249-0407$ & - & S33 W09 & 2 & A-0 & - & Type III \\
\hline 24 & 1957 & Dec. 12 & $1757-1859$ & 1806 & N15 W41 & $2+$ & B-12 & $\mathrm{Sl} / 1$ & Type II \\
\hline $25^{*}$ & 1958 & Jan. 1 & $1747-1911$ & 1800 & N19 E47 & 2 & $\mathrm{~A}-0$ & - & $\mathrm{q}$ \\
\hline 26 & 1958 & Jan. 16 & $2255-2347$ & 2306 & S15 E47 & 2 & B-15 & - & Types II, III \\
\hline 27 & 1958 & Apr. 5 & $1925-1950$ & 1935 & N44 E82 & 2 & B-48 & $\mathrm{Sl} / 1$ & $\mathrm{q}$ \\
\hline 28 & 1958 & Apr. 29 & $1128-1240$ & 1158 & N30 E50 & $2+$ & $\mathrm{AC}-0$ & $\mathrm{Sl} / \mathbf{1}+$ & $\mathrm{q}$ \\
\hline 29 & 1958 & June 23 & $0700-0850$ & 0717 & N25 E53 & $2+$ & $A-0$ & $\mathbf{S} / 2-$ & $\mathrm{q}$ \\
\hline
\end{tabular}


(Table I continued)

\begin{tabular}{|c|c|c|c|c|c|c|c|c|c|}
\hline \multirow[t]{3}{*}{ No. } & \multirow[t]{3}{*}{ Date } & & \multicolumn{4}{|l|}{$\mathrm{H} \alpha$ Flare } & \multirow{3}{*}{$\begin{array}{l}\text { Spot } \\
\text { Data }^{a}\end{array}$} & \multirow{3}{*}{$\begin{array}{l}\text { SWF }^{b} \\
(1956-69)\end{array}$} & \multirow{3}{*}{$\begin{array}{l}\text { Remarks }{ }^{\mathrm{c}} \\
(1956-1969)\end{array}$} \\
\hline & & & \multicolumn{2}{|l|}{ Time (UT) } & \multirow[t]{2}{*}{ Position } & \multirow[t]{2}{*}{ Imp. } & & & \\
\hline & & & Start End & Max. & & & & & \\
\hline 30 & 1958 & July 23 & $0518-0618$ & 0530 & S08 E39 & 2 & $\mathrm{BC}-56$ & - & \\
\hline 31 & 1958 & Aug. 30 & $1933-2052$ & $\left\{\begin{array}{l}1946 \\
2020\end{array}\right.$ & S06 W42 & $2+$ & $A-0$ & - & \\
\hline 32 & 1958 & Sept. 7 & $1639-1726$ & 1643 & S32 E18 & 2 & $\mathrm{AC}-0$ & $\mathrm{~S} / 3$ & $2800 / 145, \mathrm{q}$ \\
\hline 33 & 1958 & Sept. 12 & $0655-0740$ & 0702 & S13 E67 & 2 & $\mathrm{BC}-22$ & $\mathrm{~S} / 2$ & \\
\hline 34 & 1958 & Oct. 28 & $1505-1617$ & - & N18 W38 & 2 & $\mathrm{BC}-20$ & - & q III \\
\hline 35 & 1958 & Nov. 14 & $0036-0207$ & 0046 & S19 E51 & 3 & B-63 & $\mathrm{Sl} / 3$ & $q$ \\
\hline 36 & & Jan. 31 & $0108-0152$ & 0115 & $\mathrm{~N} 20 \mathrm{E} 03$ & 2 & B- 47 & - & $3750 / 165$ \\
\hline 37 & 1959 & Feb. 1 & $1153-1233$ & 1203 & N23 W17 & 2 & B-47 & - & $3000 / 357$ \\
\hline 38 & & Feb. 2 & $1017-1225$ & 1039 & N22 W30 & 3 & A-0 & $\mathrm{Sl} / 1$ & \\
\hline 39 & 1959 & Feb. 19 & $2032-2244$ & 2037 & N11 W10 & 3 & BC-79 & $\mathrm{Sl} / 1+$ & $2800 />200$, III \\
\hline $40^{*}$ & 1959 & June 29 & $0854-0954$ & 0908 & S20 E29 & 2 & $\mathrm{BC}-8$ & - & $\mathrm{q}$ \\
\hline 41 & 1050 & Suly 13 & $1920-2210$ & 2016 & $\mathrm{~S} 23 \mathrm{E} 48$ & 2 & B-27 & $\mathrm{Sl} / 2$ & Cont. \\
\hline 42 & & July 14 & $1400-1730$ & 1445 & S25 E39 & 3 & B-15 & $\mathrm{S} 1 / 2+$ & Cont., III \\
\hline $43^{*}$ & 1959 & Sept. 1 & $1923-2216$ & 1938 & N12 E60 & $2+$ & $\mathrm{BC}-25$ & $\mathrm{~S} 1 / 2$ & $\begin{array}{l}\text { Types II, } \\
\text { III Cont., IV }\end{array}$ \\
\hline 44 & 1959 & Dec. 11 & $0910-0957$ & - & S16 W44 & 2 & B-39 & - & \\
\hline 45 & 1960 & Jan. 8 & $1800-2050$ & 1841 & $\mathrm{~N} 27 \mathrm{~W} 13$ & 2 & $B-10$ & - & \\
\hline $46^{*}$ & 1960 & July 7 & $0200-0326$ & 0210 & N24 E12 & 2 & B-11 & $\mathrm{S} / 1$ & $\mathrm{q}$ \\
\hline $47^{*}$ & 1960 & July 8 & $2328-2410$ & 2334 & N07 W33 & $2+$ & $\mathrm{A}-0$ & - & Type II, q \\
\hline 48 & 1960 & July 11 & $0950-1200$ & 1030 & N15 W30 & 2 & B-35 & - & d \\
\hline 49 & 1960 & July 23 & $1227-1252$ & 1230 & N14 W68 & 2 & $A-0$ & - & $q$ \\
\hline 50 & 1960 & Oct. 14 & $2033-2245$ & 2058 & S25 E56 & $2+$ & $\mathrm{B}-11$ & - & Types III, IV, q \\
\hline 51 & 1960 & Nov. 6 & $1752-2030$ & 1840 & N13 E07 & 3 & $\mathrm{~B}-88$ & $\mathrm{G} / 1$ & Type II, q \\
\hline 52 & 1961 & Mar. 18 & $0735-0842$ & - & N24 E88 & 2 & $\mathrm{~A}-0$ & - & \\
\hline 53 & 1961 & May 4 & $2150-2340$ & 2213 & S10 W56 & 3 & BC-44 & $\mathrm{Sl} / 1+$ & Types II, III and IV \\
\hline 54 & 1961 & May 9 & $1540-1942$ & 1551 & N09 E12 & 2 & B -10 & - & \\
\hline 55 & 1961 & Aug. 14 & $1400-1705$ & - & S07 W69 & 2 & $A-0$ & - & $\mathrm{q}$ \\
\hline 56 & 1961 & Aug. 22 & $1037-1415$ & 1132 & N08 W12 & 2 & B-48 & - & $\mathrm{q}$ \\
\hline 57 & 1962 & May 28 & $1629-1725$ & 1640 & S16 E78 & 2 & $\mathrm{~A}-0$ & $\mathrm{~S} 1 / 1$ & Type II \\
\hline 58 & 1962 & Oct. 23 & $1642-1745$ & 1705 & N03 W70 & 2 & $\mathrm{~A}-0$ & - & Types II, III and IV \\
\hline 59 & 1963 & May 27 & $0529-0730$ & 0548 & N11 E15 & 2 & B-10 & - & \\
\hline 60 & 1963 & June 1 & $0848-0943$ & 0853 & N09 E50 & 2 & $\mathrm{~A}-0$ & - & $q$ \\
\hline 61 & 1963 & June 14 & $0620-0738$ & 0638 & N02 W00 & 2 & $\mathrm{~B}-15$ & - & \\
\hline 62 & 1965 & June 9 & $0600-0720$ & 0638 & N21 E49 & 2 & B-69 & $\mathrm{G} / 1$ & $q$ \\
\hline 63 & 1966 & May 25 & $1042-1230$ & 1116 & N28 E20 & $2 n$ & A-0 & - & $\mathrm{d}$ \\
\hline 64 & 1966 & May 28 & $1532-1830$ & 1622 & N15 W40 & $2 b$ & B-20 & $\mathrm{Sl} / 3$ & d III \\
\hline 65 & 1966 & June 27 & $2358-2520$ & 2420 & N25 W58 & $2 n$ & B-30 & - & $\mathrm{q}$ \\
\hline 66 & 1966 & July 28 & $2214-2450$ & $\begin{array}{l}2245 \\
2330\end{array}$ & N36 E33 & $3 b$ & $\mathrm{BC}-102$ & - & $\begin{array}{l}2800 / 135, \text { Types II, } \\
\text { III, Cont., } \mathrm{q}\end{array}$ \\
\hline 67 & 1966 & Nov. 14 & $1215-1335$ & 1227 & N21E75 & $2 n$ & A -0 & $\mathrm{G} / 1$ & \\
\hline 68 & 1966 & Dec. 13 & $2300-2350$ & 2310 & N24 E56 & $2 b$ & $\mathrm{BC}-40$ & $\mathrm{~S} / 1$ & Type II, $3750 / 315$ \\
\hline 69 & 1967 & Jan. 5 & $0117-0342$ & - & S26 E34 & $2 f$ & $\mathrm{AC}-0$ & - & d, Type III \\
\hline 70 & & Jan. 11 & $0131-0510$ & 0259 & $\mathrm{~S} 27 \mathrm{~W} 45$ & $3 b$ & BC-10 & - & Types II, III, d \\
\hline 71 & & Feb. 6 & $1825-1915$ & 1849 & N26E85 & $2 n$ & $\mathrm{~B}-50$ & $\mathrm{Sl} / 1-$ & Cont. \\
\hline 72 & & Feb. 13 & $1747-2130$ & 1820 & N21 W11 & $3 b$ & B-10 & $\mathrm{G} / 1+$ & Types II, III, IV, d \\
\hline 73 & 1967 & Feb. 18 & $0943-1200$ & 1035 & N22 E55 & $3 n$ & $\mathrm{BC}-\mathbf{3 0}$ & - & $\mathrm{q}$ \\
\hline 74 & & Feb. 22 & $1440-1604$ & - & N23 W01 & $2 n$ & $\mathrm{BC}-37$ & $\mathrm{G} / 1-$ & Types II, IV, q \\
\hline 75 & 1967 & Mar. 6 & $2255-2400$ & 2311 & N17 E08 & $2 n$ & $\mathrm{AC}-0$ & - & q, Type III \\
\hline
\end{tabular}


(Table I continued)

\begin{tabular}{|c|c|c|c|c|c|c|c|c|c|}
\hline \multirow[t]{3}{*}{ No. } & \multirow{3}{*}{\multicolumn{2}{|c|}{ Date }} & \multicolumn{4}{|l|}{ H $\alpha$ Flare } & \multirow{3}{*}{$\begin{array}{l}\text { Spot } \\
\text { Data }^{a}\end{array}$} & \multirow{3}{*}{$\begin{array}{l}\text { SWF }^{\mathbf{b}} \\
(1956-69)\end{array}$} & \multirow{3}{*}{$\begin{array}{l}\text { Remarks }^{c} \\
(1956-1969)\end{array}$} \\
\hline & & & \multicolumn{2}{|l|}{ Times (UT) } & \multirow[t]{2}{*}{ Position } & \multirow[t]{2}{*}{ Imp. } & & & \\
\hline & & & Start End & Max. & & & & & \\
\hline $76^{*}$ & 1967 & May 4 & $0126-0320$ & 0214 & N32 E24 & $2 n$ & B-6 & & \\
\hline 77 & & May 6 & $0435-0545$ & 0439 & S20 W34 & $3 n$ & B-27 & $\mathrm{Sl} / 2$ & \\
\hline 78 & 1967 & May 8 & $1124-1440$ & 1136 & S21 W60 & $2 n$ & B-17 & $\mathrm{S} / 1$ & $q$ \\
\hline 79 & & May 10 & $1145-1240$ & 1153 & S22 W87 & $2 n$ & $\mathrm{~A}-0$ & - & $\mathrm{q}$ \\
\hline 80 & 1967 & July 29 & $0402-0511$ & 0432 & S26 W63 & $2 n$ & $A-0$ & - & Types II, III, q \\
\hline 81 & 1967 & Sept. 17 & $0353-0420$ & 0359 & N15 E61 & $2 \mathrm{~b}$ & $A-0$ & $\mathrm{~S} / 1$ & \\
\hline 82 & 1968 & Nov. 2 & $1631-1730$ & 1700 & N19 E27 & $2 \mathrm{n}$ & $A-0$ & - & III Cont. \\
\hline 83 & 1969 & June 13 & $1549-2000$ & 1633 & S24 E68 & $3 b$ & B-50 & $\mathrm{G} / 3$ & Type II, Cont., q \\
\hline
\end{tabular}

Brackets indicate that flares were in the same center of activity.

Italization of times of flare indicates that 'start' or 'end' of flare was observed.

* Flare was located between two plages.

a In this column $\mathrm{A}$ indicates that the flare was in a spotless region; $\mathrm{B}$, in a region with a small spot; $\mathrm{C}$, in a region with a neighboring plage with significant spot within $\sim 15^{\circ}$ of flare position. The number that follows the letter gives the total area of spots in region with flare, in millionths of the hemisphere.

b This column gives data regarding Short Wave Fades. S, Sl, and G indicate sudden, slow, and gradual fades respectively. The number that follows the letter gives the importance of the SWF.

c The Remarks column gives partial information on geomagnetic disturbance and radio frequency emission. The letter $\mathrm{q}$ indicates no storm began within three days after flare; the letter $\mathrm{d}$ identifies the six flares thought to be most clearly associated with subsequent geomagnetic disturbance. Types II, III, IV or 'continuum' indicate flares associated with reports of these events on records of the dynamic spectrum. Flares with emission at centimeter wavelengths $>100 \times 10^{-22} \mathrm{Wm}^{-2}(c / s)^{-1}$ are identified by an entry that gives the frequency in $\mathrm{Mc} / \mathrm{s}$ and the peak flux in the aforementioned units.

\section{Characteristics of Flares of Importance $>2$ in Regions with Small or No Spots}

\section{A. FREQUENCY}

It has been possible to identify in worldwide data 83 instances of well observed flares of importance $>2$ in centers of activity with only small or no spots. The list is not long, but it is adequate to attest to the occurrence of major $\mathrm{H} \alpha$ flares in regions without large sunspots and to provide some indication of the characteristics of this special group of large $\mathrm{H} \alpha$ flares.

In the 11.5 years, 1957.5-1968, we know of 63 flares, importance $>2$ in regions with only small or no spots. In the same time interval, according to our reevaluation of worldwide flare data, there was a total of 925 flares of importance 2 and 3. Thus $\sim 7 \%$ of all flares of importance $>2$ in the years 1957.5-1968 occurred in centers of activity with either very small or no detectable spots. The proportion of such flares was markedly variable from year to year and ranged from only $2 \%$ in 1968 and $4 \%$ in 1957,1958 , and 1959 , to $14 \%$ in $1961,17 \%$ in 1965 , and $25 \%$ in 1967 .

\section{B. THE APPEARANCE OF THE H $\alpha$ FLARES}

We have been able to look at photographic records of 32 of the 83 flares in this study. 
This includes 12 flares of importance 3. Seventeen of the flares were recorded on films obtained at the McMath-Hulbert Observatory, and solar colleagues at CSIRO, National Standards Laboratory, Australia, and at the Mount Wilson, Kodaikanal, Meudon, and University of Hawaii Observatories have sent pictures of 15 additional cases. In each instance, the area of the flaring region is large, since the study is limited to flares evaluated as of importance 2 and 3 . The flares include examples of the three intensity classes, bright, normal, and faint. However, inspection of the photographic collection suggests that although a number of the flares are evaluated as 'bright' these flares do not appear to us to equal in intensity certain of the very brightest flares observed during the past decades, i.e., July 16, 1959, and November 12, 1960.

In general the flares in Table I were closely associated with the location of previously existing dark filaments (prominences in projection on the disk) and with motion in these filaments as indicated by Doppler shifts in the dark gases, prior to and during the occurrence of the flare. Close association between flares and filaments is not confined to flares in regions without large spots. Kiepenheuer (1963) has summarized the general relationships between these two aspects of solar activity. A number of the flares in regions without significant spots resembled the bright chromospheric phenomena described by Hyder (1967) in association with the disappearance of filaments. The dark filaments associated with the flares in Table I, either traversed a plage or lay between regions described as two plages. From reported relationships between filaments and magnetic fields (Howard, 1959) it follows that the flares in this study, like flares in regions with significant spots (Smith and Ramsey, 1967; Severny, 1958), developed on either side of the locus of zero values in the longitudinal magnetic field, the line that separates magnetic fields of opposite polarity. For the flares in spotless regions, the relevant magnetic fields would be limited to the weak fields commonly associated with $\mathrm{H} \alpha$ and $\mathrm{Ca}^{+}$plages. When the flare occurred between two plages, assignment to a single center of activity was ambiguous. Such cases are identified by asterisks in Table I. For inclusion in this study both of the associated regions were required to be free from large spots.

In most cases the flare emission began in a number of small separate areas. In certain instances this emission coalesced into two bright flare filaments, more or less parallel, which frequently showed appreciable separation with time. These bright flare ribbons either bordered the location of a previously existing filament, or lay on either side of the direction indicated by a filament (Figure 2). The occurrence of two 'parallel' bright flare filaments seems as characteristic of large $\mathrm{H} \alpha$ flares in regions with small or no spots as of certain flares in centers of activity with significantly large and complex spots. See related comments by Švestka (1968) and Švestka and Simon (1969).

Although there is abundant evidence for ejections, and for ascending and descending gases accompanying these flares, we know of no observations that would indicate the formation of a great system of loop-type prominences as part of the total flare event for the flares in Table I.

Representative examples of these large $\mathrm{H} \alpha$ flares in centers of activity with only small or no spots are shown in Figures 1 and 2. In addition, reference can be made to 
a.

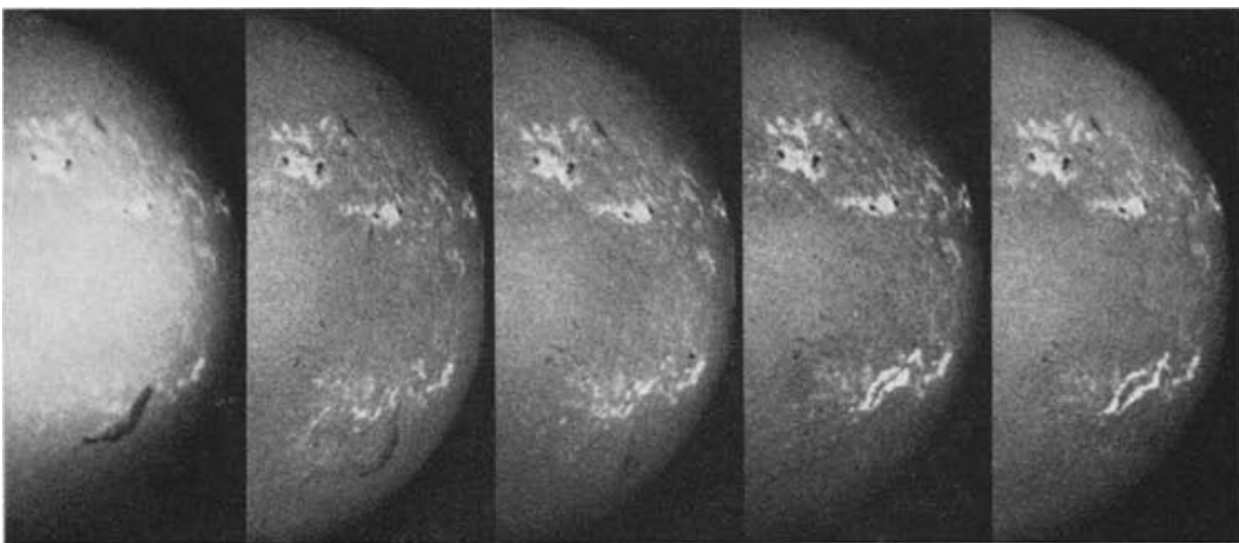

b.

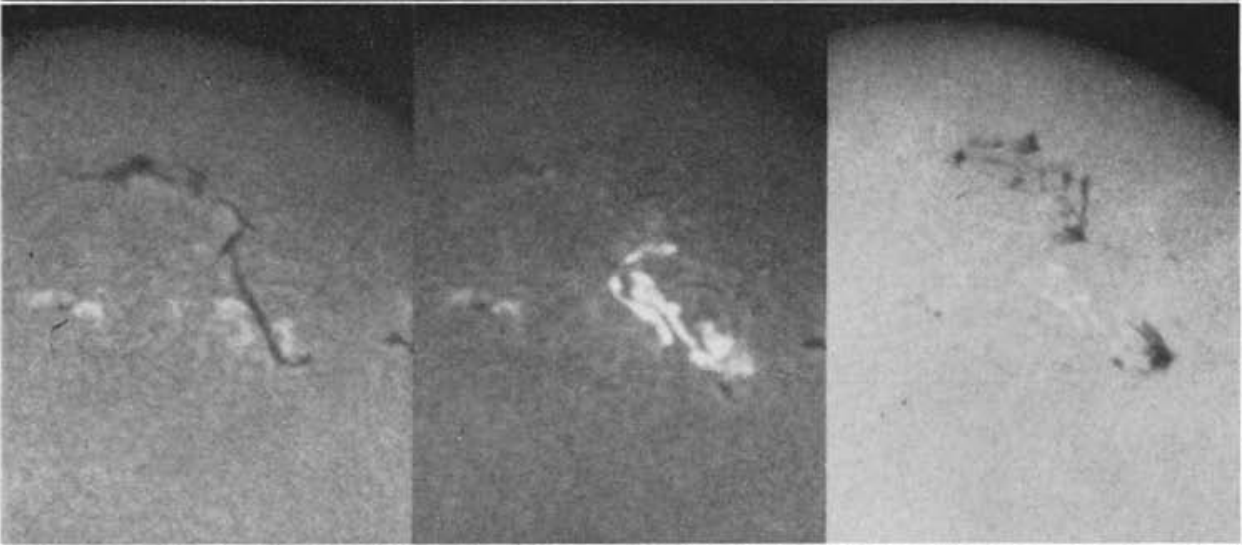

c.

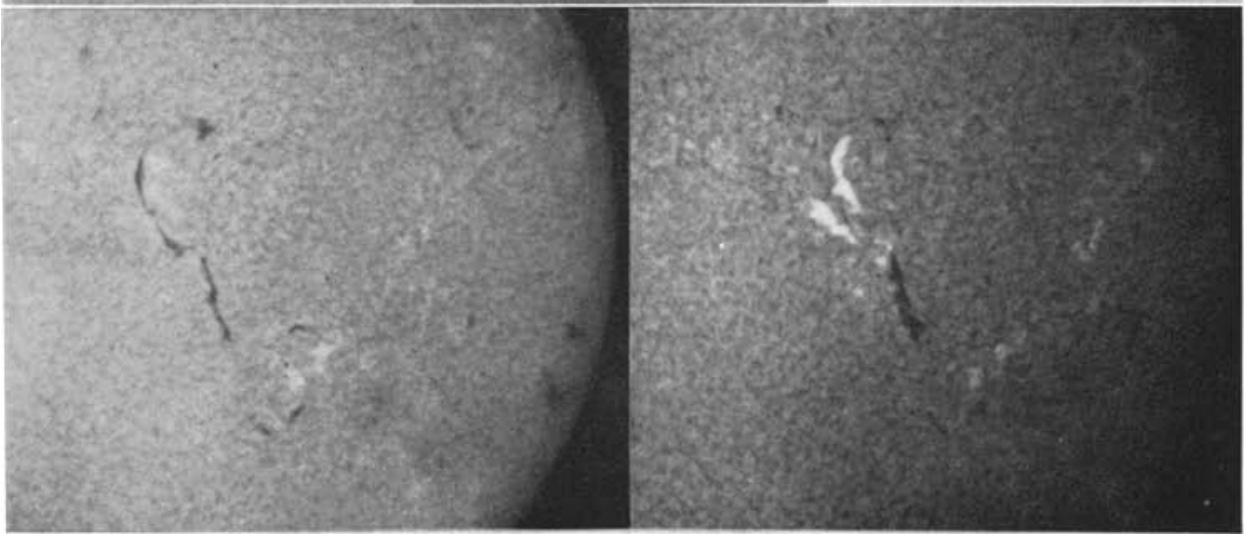

Fig. 2. $\mathrm{H} \alpha$ filtroheliograms showing relationships between previously existing dark filaments and three flares in Table I. (a) Flare No. 70: Times (UT) left to right, $1967 \mathrm{Jan} .10^{\mathrm{d}} 23^{\mathrm{h}} 05^{\mathrm{m}}, \mathrm{Jan} .11^{\mathrm{d}} 01^{\mathrm{h}} 23^{\mathrm{m}}$ $02^{\mathrm{h}} 02^{\mathrm{m}}, 02^{\mathrm{h}} 29^{\mathrm{m}}, \sim 03^{\mathrm{h}} 17^{\mathrm{m}}$. (University of Hawaii); (b) Flare No. 72: Times (UT) left to right, 1967 Feb. $13^{\mathrm{d}} 17^{\mathrm{h}} 51^{\mathrm{m}}, 18^{\mathrm{h}} 15^{\mathrm{m}}$ (cen. of $\left.\mathrm{H} \alpha\right) 18^{\mathrm{h}} 15^{\mathrm{m}}(\mathrm{H} \alpha+1 \AA)$. (McMath-Hulbert Observatory); (c) Flare No. 56: Times (UT) left to right, 1961 Aug. $21^{\mathrm{d}} 20^{\mathrm{h}} 37^{\mathrm{m}}$, Aug. $22^{\mathrm{d}} 11^{\mathrm{h}} 32^{\mathrm{m}}$. (McMath-Hulbert Observatory). 
MAJOR H $\alpha$ FLARES IN CENTERS OF ACTIVITY WITH VERY SMALL OR NO SPOTS

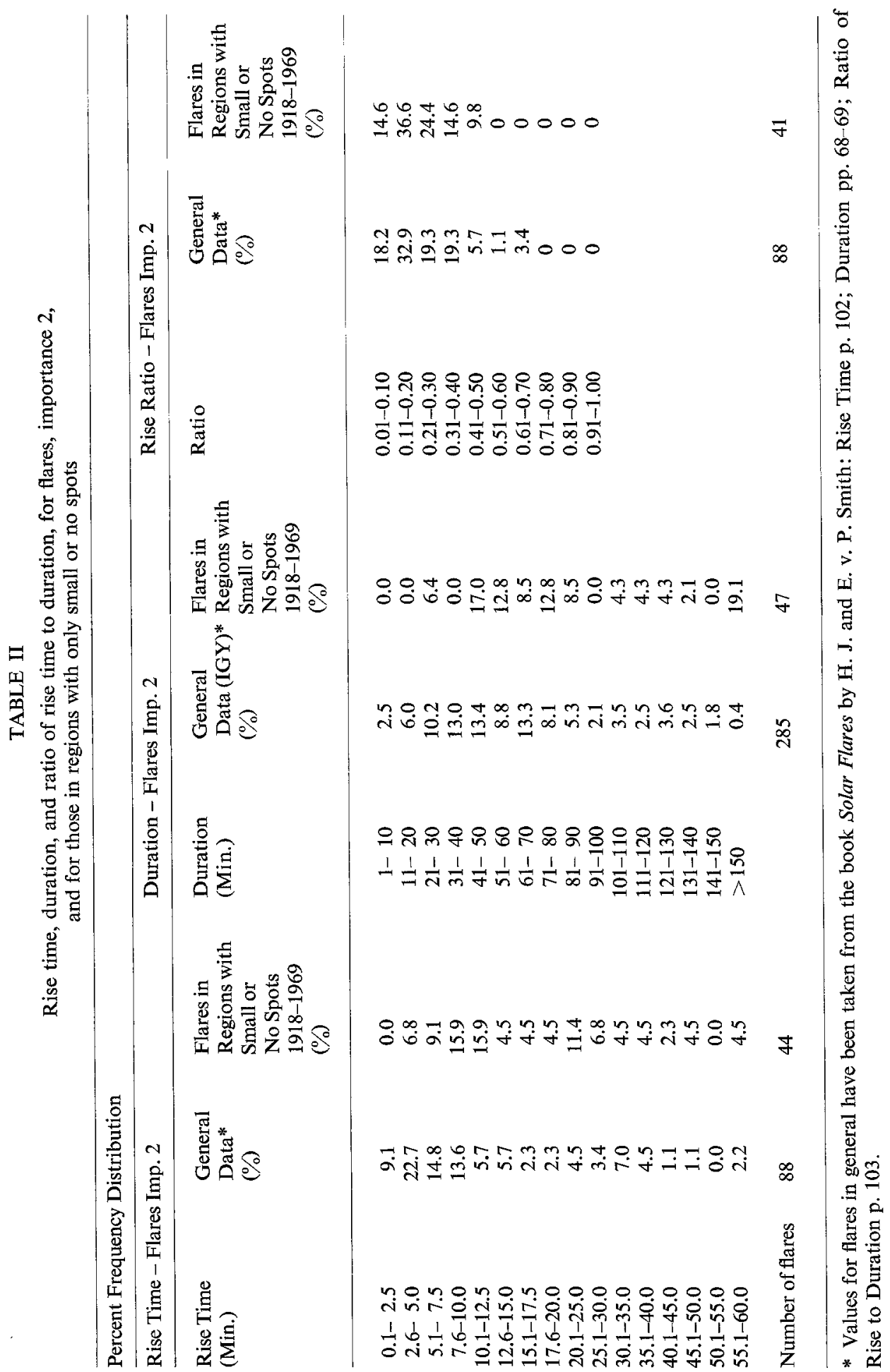


already published pictures of the flare on $1959 \mathrm{July} 14^{\mathrm{d}} 14^{\mathrm{h}} 00^{\mathrm{m}}$, by Švestka (1968), and Dodson and Hedeman (1968), and to those of the event on 1959 September $1^{\mathrm{d}} 19^{\mathrm{h}} 23^{\mathrm{m}}$ in an article by Hyder (1967).

\section{RISE-TIME AND DURATION}

According to the data in Table I, $\mathrm{H} \alpha$ flares of importance $>2$, in centers of activity with only small or no spots, rise to maximum intensity more slowly and continue longer than do flares in general with similar importance (area) evaluations. For many of these flares, a 'flash phase' appears to be absent or poorly defined. Comparison has been made with the general data on flare rise-time and duration reported by Smith and Smith (1963). (See Table II.) The relatively slow rise to maximum intensity for flares of importance 2 in regions with small or no spots is reflected in a modal rise-time of between 7.6 and $12.5 \mathrm{~min}$, compared to $2.6-5.0 \mathrm{~min}$ in the general data for flares of importance 2. A full $19 \%$ of the flares of importance 2 in regions with small or no spots continued for more than $150 \mathrm{~min}$ whereas fewer than $1 \%$ of the flares of importance 2 during the IGY lasted this length of time. There are no cases in Table I of flares with durations as short as $20 \mathrm{~min}$.

In spite of the foregoing differences in duration and time of rise to maximum intensity, the ratios of these two quantities are remarkably similar for flares of importance 2 in general and in regions with small or no spots (see Table II). All flares in Table I for which the time information is complete, rose to maximum intensity more quickly than they declined.

\section{IONIZING RADIATION}

Many of the flares in this study occurred before the monitoring of solar X-radiation. The emission of such short-wavelength, ionizing radiation, however, often can be deduced from the occurrence of a Sudden Ionospheric Disturbance (SID) on the Earth. The type of SID for which worldwide coverage has been most nearly complete in recent years is the Short-Wave Fade (SWF). We therefore have studied the occurrence of SWF's with the 69 flares of importance $>2$ in regions with small or no spots, 1956-1969, and have compared the results with similar information for all flares of importance $>2$ observed during the IGY (1957.5-1958) (Dodson and Hedeman, 1964). A summary of this comparison is shown in Table III. During the IGY, $66 \%$ of the flares of importance $>2$ were associated with confirmed SWF's. Of the 69 flares in Table I (1956-1969) only 41\% were associated with confirmed SWF's. An additional $16 \%$ were in time association with a report of some type of ionospheric disturbance. In general, however, the flares of importance 2 and 3 in regions with small or no spots were associated with a lower percentage of great SWF's (i.e. importance 2 and 3) and with a higher percentage of weak or 'no' SWF's (i.e. importance 1 or 0 ) than were correspondingly large $\mathrm{H} \alpha$ flares during the IGY. (See Table III.) It is of interest that the slow rise to maximum intensity so characteristic of the $\mathrm{H} \alpha$ flares in Table $I$ is reflected in the fact that $71 \%$ of the SWF's associated with the flares in regions without significant spots were described as 'slow' or 'gradual' rather than 'sudden.' 
The presence or absence of a neighboring center of activity with a large spot made no difference in the occurrence of SWF's with flares considered in this study.

Summarized reports of X-ray enhancements at 2-12 $\AA$ whose peak intensity is $>4$ times that of the ambient quiet Sun, have been published by Van Allen in the Solar-Geophysical Data Bulletins. (ESSA) and were available to us for the interval July 1966 - December 1968. Table IV presents a simple comparison of the time association of such $X$-radiation with confirmed flares of importance $>2$. Approximately $80 \%$ of the flares of importance $>2$ were accompanied by X-ray enhancement $(2-12 \AA)$ at least as great as 4 times the ambient quiet Sun, whether or not they were located in regions with significant spots. For X-ray bursts of greater intensity, flares in spotted

TABLE III

Percentage distribution of importance of SWF's for all $\mathrm{H} \alpha$ flares of importance $>2$ during IGY* and for $\mathrm{H} \alpha$ flares of importance $>2$ in regions with small or no spots 1956-1969

\begin{tabular}{|c|c|c|c|}
\hline $\begin{array}{l}\text { Importance } \\
\text { of SWF }\end{array}$ & \multicolumn{2}{|c|}{$\begin{array}{l}\text { Number of Flares associated } \\
\text { with SWF of Specific Importance }\end{array}$} & $\%$ of Total \\
\hline & Ha Flares, Importance 3 & & \\
\hline \multirow[t]{2}{*}{3} & IGY & 21 & $54 \%$ \\
\hline & No large spot, $1956-1969$ & 3 & 21 \\
\hline \multirow[t]{2}{*}{2} & IGY & 12 & 31 \\
\hline & No large spot, $1956-1969$ & 2 & 14 \\
\hline \multirow{2}{*}{1 and $1-$} & IGY & 1 & 3 \\
\hline & No large spot, 1956-1969 & 5 & 36 \\
\hline \multirow[t]{2}{*}{ No SWF } & IGY & 5 & 13 \\
\hline & No large spot, 1956-1969 & 4 & 29 \\
\hline \multirow[t]{2}{*}{ Total } & IGY & 39 & - \\
\hline & No large spot, $1956-1969$ & 14 & - \\
\hline
\end{tabular}

\begin{tabular}{llrr}
\hline & Ha Flares, Importance 2 & & \\
3 & IGY & 34 & $7 \%$ \\
2 & No large spot, 1956-1969 & 2 & 4 \\
& IGY & 133 & 28 \\
1 and $1-$ & No large spot, 1956-1969 & 4 & 7 \\
\multirow{2}{*}{ No SWF } & IGY & 131 & 28 \\
& No large spot, 1956-1969 & 12 & 22 \\
& IGY & 169 & 36 \\
Total & No large spot, 1956-1969 & 37 & 67 \\
& IGY & 467 & - \\
\hline Number and percent- & No large spot, 1956-1969 & 55 & - \\
age of flares of imp. & No large spot, 1956-1969 & 332 & $66^{\circ} \%$ \\
2 and 3 with SWF's. & & & $41 \%$ \\
\hline
\end{tabular}

* Based on data published by Dodson and Hedeman (1964).

+ This number increases to $57 \%$ if other types of sudden ionospheric disturbances are considered. 
TABLE TV

Comparison of number of confirmed Ha flares, imp. $>2$, July 1966 - December 1968 with associated occurrence of 2-12 $\AA$ X-ray enhancements (Van Allen data)

\begin{tabular}{|c|c|c|c|c|c|}
\hline & \multirow{3}{*}{$\begin{array}{l}\text { Number of confirmed } \\
\text { Flares Imp. }>2 \\
\text { July } 1966-\text { Dec. } 1968\end{array}$} & \multicolumn{4}{|c|}{ Associated X-ray Burst } \\
\hline & & \multicolumn{2}{|c|}{$>4 \times$ Quiet Sun } & \multicolumn{2}{|c|}{$\$ 20 \times$ Quiet Sun } \\
\hline & & Number & Percent & Number & Percent \\
\hline $\begin{array}{l}\text { In regions without } \\
\text { large spots }\end{array}$ & $14^{\mathrm{a}}$ & 11 & $78 \%$ & 2 & $14 \%$ \\
\hline $\begin{array}{l}\text { In regions with } \\
\text { large spots }\end{array}$ & $103^{b}$ & 84 & 81 & 28 & 27 \\
\hline Total & $117^{\mathrm{c}}$ & 95 & $81 \%$ & 30 & $26 \%$ \\
\hline
\end{tabular}

${ }^{a}$ Excludes 3 cases at times of no X-ray data.

b Excludes 10 cases at times of no X-ray data.

- Excludes 13 cases at times of no X-ray data.

regions appear to be favored. There are only two flares in Table I (July 1966 - December 1968) for which the X-ray flux (2-12 $\AA$ ) was observed to be as great as 20 times that of the quiet Sun, that of 1967 May 6 with a flux of 74 and that on 1967 May 10 with a peak of 20. Both of these flares took place in McMath plage 8791. On May 6 the area of the associated spot was only 27 millionths and on May 10 no spot was observed.

A paper by Walker and Rugge (1968) describes the X-ray spectrum below $25 \AA$ during the flare of 1967 February 13 (No. 72 in Table I). They report that in comparison to spectra obtained during times of only moderate solar activity, lines of low ionization potential increased $50 \%$, and lines of higher ionization potential (FexvII, Nex, MgXI, Fexvm, and Nixix) increased in intensity from three to ten times. In addition, lines with wavelengths appropriate to transitions of FexIx through FexxIV were observed. Apparently very high excitation phenomena can occur during large $\mathrm{H} \alpha$ flares even when, as on 1967 February 13 the spot, if visible at all, is no greater in area than 10 millionths of the solar hemisphere.

The X-ray emission associated with the major flares in regions with small or no spots shared the characteristic slow rise to maximum intensity exhibited by the $\mathrm{H} \alpha$ flare emission itself. For none of the flares in Table I did the X-radiation reach peak flux in as short a time as ten minutes, whereas for one-third of the X-ray bursts associated with flares of importance $>2$ in regions with larger spots, the time for rise to maximum was $₹ 10 \mathrm{~min}$.

\section{E. RADIO FREQUENCY EMISSION}

Radio frequency emission at $\sim 10 \mathrm{~cm}$ accompanied a large proportion of the flares in Table I that occurred during the hours when this emission was being recorded. In general the maximum flux was low. In order to evaluate these circumstances, a comparison has been made of $\sim 10 \mathrm{~cm}$ emission accompanying flares of importance $>2$ 
in regions with, and without, significant spots for the years 1966-1968 inclusive. Initially, the comparison is limited to flares that occurred during the observing hours of the radio telescopes of the National Research Council of Canada. Of the 61 flares of importance $>2(1966-1968)$ in regions with significant spots for which the Canadian radio telescopes provide complete data, $92 \%$ were accompanied by long-enduring $10 \mathrm{~cm}$ emission of the type described as 'rise and fall' or 'post burst increase'. In addition, $93 \%$ of these flares also were associated with a simple or complex impulsive burst. The same radio emission records cover the occurrence of 11 flares of importance $>2$ in regions with only small or no spots. All of these flares were accompanied by long-enduring radiation, but for only 6 were impulsive bursts reported.

The same pattern of emission is maintained if the radio-frequency data for flares in regions without major spots is extended to include worldwide observations in the neighborhood of $10 \mathrm{~cm}$ for the years 1966-1968. The number of flares of importance $\$ 2$ in regions with small or no spots increases to 20 . Again, all such flares were accompanied by long-enduring radiation, but only 9 , or $45 \%$, were accompanied by impulsive bursts.

Maximum values of the peak flux of the long-enduring radiation that accompanied major $\mathbf{H} \alpha$ flares in regions with and without significant spots appear to be similar. For impulsive bursts this is not the case. Impulsive bursts tend to be much stronger as well as more frequent with major $\mathrm{H} \alpha$ flares in regions with significant spots than for such flares in regions with only small or no spots. We know of only one flare in Table $\mathbf{I}$ for which the flux at $\sim 10 \mathrm{~cm}$ was as great as $500 \times 10^{-22} \mathrm{Wm}^{-2}(c / s)^{-1}$ (1956 February $\left.29^{\mathrm{d}} 22^{\mathrm{h}} 20^{\mathrm{m}}\right)$, but during $1966-1968$ on the records of the Canadian radio telescopes such bursts accompanied more than $19 \%$ of the flares of importance $>2$ in regions with significant spots. The physical circumstances in a center of activity that lead to the presence or absence of a major spot appear to have but little effect on those processes, presumably thermal, that are associated with the emission of "long-enduring' flare-associated radiation at $10 \mathrm{~cm}$. On the other hand, either the absence of a significant spot in a region with a major $\mathrm{H} \alpha$ flare, or the nature of flares in such regions, appears to diminish markedly the chance of occurrence of a very strong impulsive burst at these wavelengths.

Dynamic spectrum events at radio frequencies as reported in the Solar-Geophysical Data Reports and in the Quarterly Bulletin on Solar Activity have been examined for the 69 flares of importance $>2$ in regions with small or no spots that we have listed for 1956-1969. Thirty-one of these flares occurred when observations of the dynamic spectrum were not being obtained. Of the remaining 38 flares, $68 \%$ were in time-association with a reported event in the dynamic spectrum. All types of events are represented and they occurred variously in the metric and dekametric ranges. Type IV or continuum radiation accompanied 12 of the flares and Type II bursts were recorded in time association with 16 of the 38 flares for which observations of the dynamic spectrum are available.

An attempt has been made to compare the occurrence of type II bursts with major flares in regions with and without significant spots. According to our reevaluation of 
flare data, there were 637 'confirmed' flares of importance $>2$ from 1958-1963 inclusive. Of these flares, 114 or $18 \%$ were accompanied by reported Type II bursts. It must be remembered that the worldwide patrol for dynamic spectrum data is not complete for the Universal Day and that therefore the above percentage of association between major flares and the occurrence of Type II bursts is too low. In the years 1956-1969, there were 69 flares of importance $>2$ in regions without significant spots. Of these flares 16 , or $23 \%$, were accompanied by Type II bursts. Insofar as the data and coverage are comparable, for the two groups of flares, the figures suggest that the occurrence of a Type II burst with a major $\mathrm{H} \alpha$ flare was not hindered by the absence of a major spot, nor by the relatively slow rise to maximum intensity of the $\mathrm{H} \alpha$ flareemission which characterizes major flares in regions without large spots. As with flares in general, the Type II bursts associated with the flares in Table I began well after the start of the $\mathrm{H} \alpha$ flare and the reported beginning of the Type II burst was often close to or just after the time of maximum $\mathrm{H} \alpha$ intensity.

Radioheliograms obtained in Australia and recent studies by Wild (1968), have indicated that instabilities and certain flare-like phenomena may be triggered by shock waves or other phenomena associated with Type II bursts. Since the flares of Table I are rare and unusual events, we have examined the data for the possible occurrence of 'triggering' Type II bursts prior to their onset. In our judgment, we found no evidence that the flares of Table I were initiated by this mechanism. For $\frac{1}{3}$ of the flares in Table I (1956-1969) more than a week had elapsed between an observed Type II burst and the start of the flare in question. For only 9 of the 69 flares examined had a Type II burst been observed during the preceding $24 \mathrm{~h}$, and in only 3 of these cases (Nos. 44, 61 and 82) was the intervening interval less than $10 \mathrm{~h}$. (It must be remembered that observations in the dynamic spectrum do not cover the entire Universal Day.)

\section{Characteristics of Regions without Large Spots in which Major $\mathbf{H} \alpha$ Flares Have Occurred}

This study has been concerned with the occurrence of large (importance $>2$ ) $\mathrm{H} \alpha$ flares in plages with small or no spots and with a comparison between these events and similarly large $\mathrm{H} \alpha$ flares in centers of activity with much larger photospheric spots. Since the absence of a large spot may reflect only a certain phase in the development of a center of activity, it seems appropriate to examine further the characteristics of the regions in which the flares listed in Table I of this study took place.

At the time of the occurrence of the flares in Table I, the calcium plages in which the flares were located, generally were not new regions in their first transit of the solar disk. For 63 of the 69 flares in Table I from 1956-1969, the positions of the flares led clearly to locations within specific centers of activity. Of these centers of activity, $46 \%$ were in their second rotation, $43 \%$ were 3-8 rotations old, and only $11 \%$ were considered to be plages in their first rotation. Six flares were located near the position of a filament that lay between two features of the chromosphere, each of which had been 
described in monthly summaries of solar data as separate calcium plages. In these cases the two adjacent plages generally were the returning aspects of a single center of activity in an earlier rotation. The flares that occurred in this manner between such centers of activity are identified by asterisks in Table I.

In the rotations following the occurrence of the flares listed in Table $\mathrm{I}$, the associated calcium plages generally declined in size, brightness, and flare activity. Major $\mathbf{H} \alpha$ flares in regions with only small or no spots thus appear to be events in the late or dying phase of certain centers of activity. Although small spots sometimes formed on the day following certain of the flares in Table $\mathrm{I}$, there is no indication that the occurrence of a major flare in a relatively spotless region presaged a general resurgence of activity in the region. Instead, the regions in which the flares of Table I occurred generally declined further in size and brightness, or disappeared entirely, before the next rotation.

Centers of activity, after the photospheric spots have become very small or have disappeared, apparently can produce more than one large (important) $\mathrm{H} \alpha$ fiare. Table I includes 7 instances of 2 or 3 major $\mathrm{H} \alpha$ flares in the same center of activity. These cases are indicated by brackets in Table I. There is even one case in which the same spot-poor center of activity was the site of two of the flares in Table I (No. 67 and 68) in successive rotations. Therefore it appears that certain centers of activity can produce several large $\mathrm{H} \alpha$ flares in time intervals that cover at least two rotations during the spot-poor, declining phase of the region.

Many of the plages in which the flares of Table I occurred were remnants or late aspects of centers or zones of activity which, in prior rotations, had been the sites of many flares. For 28 of the 68 flares in Table I (1956-1968) the associated plage apparently was a direct return of a center of activity that in prior rotations had been flarerich. In 16 additional cases, the plage in question was a return of a region that had formed close to the perimeter of a major flaring center of activity and is considered by us as being part of a flare-rich 'zone' of activity larger than a single plage. An earlier study already has pointed out the development of 'isolated major flares' in the late history of centers of activity associated with flares producing ground-level enhancements of cosmic rays (Dodson and Hedeman, 1969).

Table I lists 24 flares (1956-1968) in 17 plages for which there is no prior history of flare-richness on the visible hemisphere. These cases include the major, southern flares of 1959 July 13 and 14 and those of 1967 February 6 and 13 . For certain of these cases an interval of flare-richness may have occurred while the plage in question was on the invisible hemisphere as is argued for the flares on 1967 February 6 and 13 (Dodson and Hedeman, 1969). Solar records show that a center of activity often passes through its interval of abundant flaring in less than 13 days. For other cases, the connection, if any, with a prior flare-rich center of activity is more remote. Four flares in Table I occurred close to a filament that had persisted for many rotations since its origin in or near a major center of activity. In these cases, the large $\mathrm{H} \alpha$ flare occurred in the remnants of the plages and filaments that had drifted poleward and to decreasing longitudes in the 4-8 rotations following the occurrence of a major zone 
of flare activity. In tracing these associations we were guided by identification of the filaments on the Cartes Synoptiques (Meudon).

Study of the regions associated with the flares of Table I further shows that, in general, major $\mathrm{H} \alpha$ flares in plages without significant spots occurred at times when the center of activity in question was not producing many flares, important or unimportant. Eleven of the flares in Table I were the only flares observed in the respective plages during their transit of the solar disk. For 13 other cases, only one other flare was reported in the associated plage. Of the regions associated with the flares in Table I (1956-1969) only $23 \%$ were the sites of more than 7 flares during the transits in which the flares in Table I took place. Accordingly, many of the major flares in regions with small or no spots can be described appropriately as 'isolated major flares'.

It should be pointed out, however, that Table I contains two marked exceptions to the preceding general description of the centers of activity without significant spots in which major flares developed. These important exceptions are described below.

(a) The flares of 1959 January 31, February 1, and February 2 occurred in McMath Plage No. 4983, a relatively flare-rich region that returned even more flare-rich in subsequent rotations. At the time of the above flares, the center of activity was in its second rotation. It had been the site of only 4 flares during its first transit of the solar disk. During its second transit in late January and early February, the region included a rapidly diminishing spot which was not visible after February 1. During this transit, 19 flares of importance $>1$ occurred in the region, 3 of which were of importance $>2$ and are included in Table $I$. In the next rotation in late February, this region returns as McMath No. 5026 and undergoes a resurgence in activity on the disk with newly formed spots and 34 flares. In March the region returned as No. 5061 with 21 flares, and in April as No. 5102 with 5 flares. After this date flare activity in this zone of the Sun diminished for several months, but was soon resumed when this solar zone developed into the unusual flare and particle-producing center at N16 $6^{\circ}$ with central meridian passage July 14, 1959.

(b) In general, the spots in the plages associated with the flares in Table I were not only small or absent on the day of the flare, but they usually were small or of only moderate size throughout the entire disk transit of the region. For the flare of 1961 May 4 (No. 53) the circumstances were different. The plage in which this flare occurred had been associated with spots with area $>500$ millionths of the hemisphere in the earlier part of its transit. The area of the spots was decreasing rapidly from east limb passage onward. On May 4 total spot area was only 44 millionths of the hemisphere and the spots disappeared on May 5. During the May transit of the solar disk this region was flare-rich with 30 flares of importance $>1$ within its bounds. All but 3 of the flares occurred prior to May 2 while the spot area exceeded 200 millionths of the hemisphere.

\section{Particle Effects of Major $\mathrm{H} \alpha$ Flares in Regions with Small or No Spots}

In general, flares of importance $\$ 2$, in regions with small or no spots, as exemplified by the flares in Table I, were not associated with subsequent detection of high-energy 
particles in the neighborhood of the Earth. A small number of exceptions to this statement may have occurred. Polar cap absorption, albeit small, was recorded in appropriate time association with the flare on 1967 February $13^{\mathrm{d}} 17^{\mathrm{h}} 47^{\mathrm{m}}$ (Masley and Goedeke, 1968). Malitson (1963) lists a small PCA on September 2, 1959 in the hours following flare No. 43 in Table I. Satellite data provide additional instances of possible proton emissions. Bryant et al. (1965a) report observation of protons by Explorer XIV following the flare of 1962 October $23^{\mathrm{d}} 16^{\mathrm{h}} 42^{\mathrm{m}}$, and in another publication the same authors (1965b) show a proton event following the flare of $1963 \mathrm{June} 14^{\mathrm{d}} 06^{\mathrm{b}} 20^{\mathrm{m}}$. Lin and Anderson (1967) associate a delayed electron and proton event with the flare on $1966 \mathrm{July} 28^{\mathrm{d}} 22^{\mathrm{h}} 14^{\mathrm{m}}$. Apparently the absence of a significant spot in a region in which a large $\mathrm{H} \alpha$ flare occurs represents circumstances which are unfavorable, but not entirely prohibitive, to the acceleration of energetic particles.

Emission of solar plasma and the particles of lower energy associated with the onset of geomagnetic storms, likewise, is not a general characteristic of the flares of Table I. A study of the onset of geomagnetic storms subsequent to the occurrence of these flares fails to provide convincing evidence that large numbers of them played a significant role in causing geomagnetic disturbances. For 31 of the 69 flares in Table I (1956-1969) geomagnetic records were quiet or without new disturbances during the following three days. These flares are identified by the letter ' $q$ ' in the Remarks column of Table I. For the remaining 38 flares geomagnetic storms began within three days. In 32 of these cases there were alternative flares as the possible source of the particles, or the storms themselves were in a pattern of 27-day recurrence.

There were, however, 6 well defined geomagnetic storms for which flares in Table I represent what seems to be the most obvious source of the storm-producing particles, if flares are considered to be the source of such particles. These storms began on 1960 July 14, 1966 May 26, 1966 May 31, 1967 January 7, 1967 January 13, and 1967 February 16. The flares associated by us with these geomagnetic disturbances are identified by the letter 'd' in the Remarks column of Table I. For none of the storms do we know of alternative, prior major flares in regions with significant spots to which the particle emission can be attributed readily, and the storms were not sequential.

Detailed studies of the interplanetary medium prior to and during two of the six storms mentioned above already have been published. In these studies Bame et al. (1968) and Hirshberg et al. (1969) have described their extensive observations of the solar wind on 1967 January 13-14 and 1967 February 15-16, and they ascribe the phenomena to two flares in Table I, Nos. 70 and 72 respectively, In both of these events, the helium to hydrogen ratio in the solar wind was markedly enhanced. Pictures of the two flares apparently responsible for these major interplanetary phenomena are shown in Figure 2. In each case, the $\mathrm{H} \alpha$ flare was closely associated with the disappearance of a very large filament.

If the above-mentioned geomagnetic storms and interplanetary phenomena were indeed caused by particles emitted at the time of the suggested flares, it would appear that major $\mathrm{H} \alpha$ flares in spot-poor centers of activity are associated occasionally with major ejections of solar plasma. 


\section{Summary}

(1) A search of flare and spot data has provided information on the occurrence of 83 flares of importance $>2$ in regions with only small or no spots, 1918-1969.

(2) Relatively complete information for 1956-1969 indicates that these rare major flares represented $\sim 7 \%$ of all confirmed flares of importance $>2$ in those years.

(3) On monochromatic pictures the large $\mathrm{H} \alpha$ flares in regions with small or no spots resembled, in general, other large $\mathrm{H} \alpha$ flares and included a number of cases of two bright flare-filaments, more or less parallel. Available photographic records did not lead to the recognition of the formation of systems of loop-type prominences in association with any of the flares.

(4) The major flares in regions without large spots were closely associated with the location of dark filaments and with motions of the gases in these filaments.

(5) The major flares in regions without significant spots rose to maximum intensity more slowly and continued longer than did major flares in general. Frequently they were flares without a well defined flash phase.

(6) Only $41 \%$ of the flares of importance $>2$ in regions with small or no spots, 1956-1969, were accompanied by confirmed short-wave fades, and only a small proportion of these ionospheric disturbances were great events.

(7) For flares of importance $>2$, July 1966 - December 1968, the presence or absence of a significant spot did not affect the frequency of occurrence of associated Xray enhancements $(2-12 \AA)>4$ times the ambient quiet Sun. For X-ray bursts $\$ 20$ times the quiet Sun, major flares in spotted regions were favored.

(8) The presence or absence of a significant spot did not affect the frequency of occurrence or the magnitude of long-enduring $\sim 10 \mathrm{~cm}$ radiation (gradual rise and fall and post burst increase) in time association with major $\mathrm{H} \alpha$ flares. On the other hand, the occurrence of strong impulsive bursts at $\sim 10 \mathrm{~cm}$ in time association with such flares was greatly reduced if spots were not present in the center of activity. The frequency of Type II bursts seemed independent of the absence or presence of significant spots in the flaring region.

(9) The flares of importance $\$ 2$ in regions with small or no spots developed in general, during the late, flare-poor stages of a center of activity or solar zone which in earlier rotations had been associated with extensive flaring. With a few exceptions the occurrence of these isolated major flares did not presage a general resurgence of activity in the region in subsequent rotations.

(10) In general, major $H \alpha$ flares in regions without significant spots were not associated with the subsequent detection of high-energy particles or the onset of geomagnetic disturbance. There were, nevertheless, a small number of exceptions to this statement which indicate that the presence of a large complex spot apparently is not necessary for the acceleration of energetic particles or the emission of solar plasma at the time of a large $\mathrm{H} \alpha$ flare.

Apparently an $\mathrm{H} \alpha$ flare is a chromospheric phenomenon that can occur throughout much of the long life of a center of activity. When a center of activity includes large 
or complex spots, flares are frequent and are often associated with energetic phenomena, but major $\mathrm{H} \alpha$ flares are not confined to this stage of development of a region. The large $\mathrm{H} \alpha$ flares that occur occasionally in plages with only small or no spots emphasize the relative simplicity of solar circumstances that can lead to the occurrence of these remarkable events.

\section{Acknowledgements}

The authors are most grateful to colleagues at the Mount Wilson, Kodaikanal, University of Hawaii, and Meudon Observatories and at CSIRO in Sydney for their interest and cooperation in graciously sending pictures of certain of the flares that formed the basis of this study. The work was supported in part by funds from the following grants and contracts: NASA NGL-23-005-275, Nonr.-1224(19), N0001467-A-0181-0001, and NSF GA-632.

\section{References}

Bame, S. J., Asbridge, J. R., Hundhausen, A. J., and Strong, I. B.: 1968, J. Geophys. Res. 73, 5761. Bryant, D. A., Cline, T. L., Desai, U. D., and McDonald, F. B.: 1965a, Astrophys. J. 141, 478.

Bryant, D. A., Cline, T. L., Desai, U. D., and McDonald, F. B.: 1965b, Phys. Rev. Letters 14, 481. Bruzek, A.: 1959, Physikalische Verhandlungen 10, 230.

Cartes Synoptiques de la Chromosphère Solaire: Published by Observatoire de Paris, Section d' Astrophysique, à Meudon.

Dodson, H. W. and Hedeman, E. R.: 1964, Planetary Space Sci. 12, 393.

Dodson, H. W. and Hedeman, E. R.: 1968, in 'Mass Motions in Solar Flares and Related Phenomena', Nobel Symposium 9, 37.

Dodson, H. W. and Hedeman, E. R.: 1969, Solar Phys. 9, 278.

Giovanelli, R. G.: 1939, Astrophys. J. 89, 555.

Hirshberg, J., Alksne, A., Colburn, D. S., Bame, S. J., and Hundhausen, A. J.: 1970, J. Geophys. Res. 75, January 1, 1970 (in press).

Howard, R.: 1959, Astrophys. J. 130, 193.

Hyder, C. L.: 1967, Solar Phys. 2, 49.

Kiepenheuer, K. O.: 1963, in AAS-NASA Symposium on the Physics of Solar Flares, 323.

Lin, R. P. and Anderson, K. A.: 1967, Solar Phys. 1, 446.

Malitson, H.: 1963, in Solar Proton Manual (ed. by F. B. McDonald), Pub. NASA-GSFC Report No. X-611-62-122.

Masley, A. J. and Goedeke, A. D.: 1968, Can. J. Phys. 46, 766.

Newton, H. W.: 1935, Monthly Notices Roy. Astron. Soc. 95, 650.

Quarterly Bulletin on Solar Activity, published for the International Astronomical Union by the Eidgen. Sternwarte, Zürich.

Richardson, R. S.: 1944, Publ. Astron. Soc. Pacific 56, 161.

Severny, A. B.: 1958, Izv. Krymsk. Astrofiz. Observ. $20,22$.

Smith, H. J. and Smith, E. v. P.: 1963, Solar Flares, Macmillan Co., New York and CollierMacmillan Ltd., London.

Smith, S. F. and Ramsey, H. E.: 1967, Solar Phys. 2, 1958.

Solar-Geophysical Data Bulletins: Published monthly by Environmental Science Services Administration, U.S. Dept. of Commerce, Boulder, Colo.

Švestka, Z.: 1968, in 'Mass Motions in Solar Flares and Related Phenomena', Nobel Symp. 9, 17.

Švestka, Z. and Simon, P.: 1969, Solar Phys. 10, 3.

Walker, A. B. C. and Rugge, H. R.: 1968, Paper 'Enhancement of the Solar X-Ray Spectrum below $25 \AA$ during Solar Flares,' COSPAR, Plenary Meeting, 11th, Tokyo, May 9-21, 1968.

Wild, J. P.: 1968, in Proceedings of Conference on 'Plasma Instabilities in Astrophysics', Asilomar, Cal. (ed. by D. A. Tidman and D. C. Wentzel), p. 119. 